\title{
Ferromagnetism, glassiness, and metastability in a dilute dipolar-coupled magnet
}

\author{
D. H. Reich and T. F. Rosenbaum \\ The James Franck Institute and Department of Physics, \\ The University of Chicago, Chicago, Illinois 60637 \\ G. Aeppli and H. J. Guggenheim \\ AT\&T Bell Laboratories, Murray Hill, New Jersey 07974
}

(Received 30 July 1986)

\begin{abstract}
We have measured the ac magnetic susceptibility of the model dilute dipolar-coupled Ising system $\mathrm{LiHo}_{x} \mathrm{Y}_{1-x} \mathrm{~F}_{4}$. The $x=0.46$ material displays an ordinary ferromagnetic transition, while the $x=0.045$ and 0.167 samples are two very different magnetic glasses. Thermal relaxation times are more than five times longer for $x=0.167$ than for $x=0.045$. In addition, the more concentrated glass shows history dependence and metastability upon field cooling.
\end{abstract}

Determining the ground-state configuration of a large number of interacting dipoles located at particular sites in a regular crystalline lattice is a seemingly simple proposition. Nonetheless, it is difficult to solve this fundamental physical problem because of the anisotropy and long-range nature of the dipolar interaction. Indeed, it is even nontrivial to show that there are ordered lattices for which the ground state is ferromagnetic. ${ }^{1}$ It should come as no surprise, therefore, that almost nothing is known about the ground state of dipoles occupying randomly chosen sites of a regular lattice. The only theoretical work of which we are aware is described in two papers ${ }^{2,3}$ concerned with the limits where the fraction $x$ of occupied sites approaches zero and unity, respectively. The former contains the interesting speculation that a spin-glass phase might exist for $x<x_{c}$. Because no experiments to date have even tested this speculation, let alone probed what may occur for $x \gtrsim x_{c}$, we have carried out a study of the dilution series $\mathrm{LiHo}_{x} \mathrm{Y}_{1-x} \mathrm{~F}_{4}$, where the rare-earth sites are randomly occupied by either magnetic $\mathrm{Ho}^{3+}$ or nonmagnetic $\mathrm{Y}^{3+}$ ions. This series has many properties which make it ideal for the study of randomly situated dipoles. The compounds are insulating and isostructural, and the lattice constants vary by less than $0.01 \%$ in going from $x=0$ to $x=1 .{ }^{4}$ Chemical segregation effects can be ruled out explicitly by the narrowness of the transition width in neutron scattering studies. ${ }^{5}$ The Ising-like $\mathrm{Ho}^{3+}$ moments are large $\left(\mu_{\mathrm{eff}}=7 \mu_{B}\right)$ and far apart so that the dipolar interaction is large compared to even the nearest-neighbor exchange interactions. For $x=1$, they order ferromagnetically at $T_{c}=1.53 \mathrm{~K}$, which is well below $\Delta=10 \mathrm{~K}$, the energy associated with the single-ion Ising anisotropy. ${ }^{6}$

The long-ranged coupled ferromagnets $\mathrm{LiHoF}_{4}$ and $\mathrm{LiTbF}_{4}$ have received considerable attention because the upper marginal dimension beyond which their critical behavior becomes mean-field-like is three, instead of four, as it is for short-ranged coupled systems. ${ }^{7}$ Inspired by predictions of novel critical behavior ${ }^{3}$ in slightly diluted dipolar-coupled ferromagnets, various investigators ${ }^{8}$ studied the $\operatorname{LiTb}_{x} \mathrm{Y}_{1-x} \mathrm{~F}_{4}$ series. Unfortunately, due to the singlet nature of the ground-state wave function for an isolated $\mathrm{Tb}^{3+}$ ion in the body-centered tetragonal $\mathrm{LiRF}_{4}$
$\left(R=\right.$ rare earth) structure, pure $\mathrm{LiTbF}_{4}$ is an induced moment ferromagnet, ${ }^{9}$ and the interesting magnetic properties of the dilution series are manifestations of crossover to Van Vleck paramagnetism rather than direct consequences of randomness. This is not the case for $\mathrm{LiHo}_{x} \mathrm{Y}_{1-x} \mathrm{~F}_{4}$ because the ground state of the $\mathrm{Ho}^{3+}$ ion is a Kramers doublet. ${ }^{6}$

Our $\mathrm{LiHo}_{x} \mathrm{Y}_{1-{ }_{x}} \mathrm{~F}_{4}$ samples were typically $0.5 \times 0.5 \times 1.0$ $\mathrm{cm}^{3}$ in size, had masses of order $1.0 \mathrm{~g}$, and were cut with the Ising (c) axis parallel to their lengths. The holmium concentration of each sample was determined simply from its density, which we measured to $\pm 0.05 \%$, using an airwater differential weighing technique. We measured $\chi(T)=\chi^{\prime}+i \chi^{\prime \prime}$ between 0.01 and $0.90 \mathrm{~K}$ in a dilution refrigerator, using a mutual inductance bridge ${ }^{10}$ transformer coupled to a PAR 124A lock-in amplifier. A potentiometer reduced the voltage developed by the standard mutual inductance until bridge balance was achieved. The inductive response was linear in the ac excitation field, which was limited to $3.2 \times 10^{-3}$ Oe to avoid heating. No effort was made to compensate for the earth's magnetic field.

Figure 1 shows the real and imaginary parts of $\chi(T)$ at frequency $f=50 \mathrm{~Hz}$ for $x=0.460,0.167$, and 0.045 . The vertical scales were calibrated by fitting $\chi^{\prime}$ to a Curie law at high $T$, using a moment of $7 \mu_{B}$ per $\mathrm{Ho}^{3+}$ ion. The $x=0.46$ sample [Fig. 1(a)] behaves like the pure ferromagnet. As $T$ is decreased, $\chi^{\prime}$ increases to the demagnetizing limit ${ }^{11}$ at $T_{c}=0.685 \mathrm{~K}$, and subsequently decreases again, as expected for Ising systems. $\chi^{\prime \prime}$ also peaks at $T_{c}$, but is considerably smaller than $\chi^{\prime} . T_{c}$ satisfies the mean-field prediction, $T_{c}(x)=x T_{c}(x=1)$, to within the errors in measuring temperature and concentration. There are no long-time relaxation processes at temperatures below the peak in $\chi$ (in contrast to the more diluted samples) and we can exclude either Arrhenius or VogelFulcher dependence of the peak position on frequency. The small rise in $\chi^{\prime \prime}$ below $T=0.1 \mathrm{~K}$ may signal reentrant behavior into a spin-glass state. ${ }^{12}$

The $x=0.167$ sample [Fig. 1(b)] is very unconventional when compared to the $x=0.460$ material. First, the real and imaginary parts of the susceptibility are of comparable magnitude, which indicates that magnetic relaxation in 


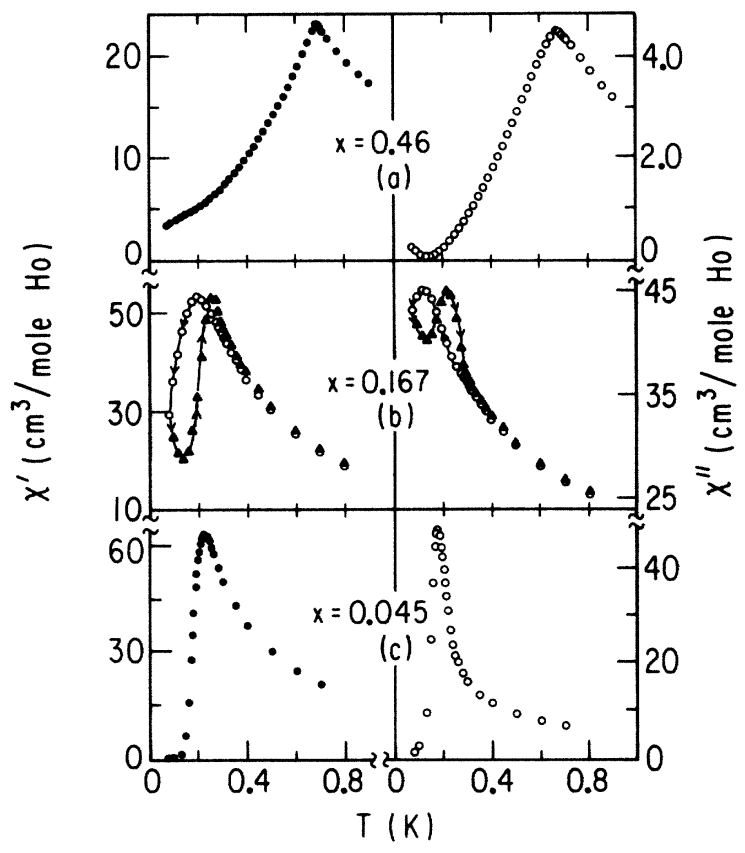

FIG. 1. The real and imaginary parts of the ac magnetic susceptibility at $f=50 \mathrm{~Hz}$ for $\mathrm{LiHo}_{x} \mathrm{Y}_{1-x} \mathrm{~F}_{4}$. Data for (a) and (c) are equilibrium values. Open circles (filled triangles) are cooling (warming) data in (b), where equilibration time is of order a day per point. The $x=0.46$ sample appears to be a conventional ferromagnet, the $x=0.167$ and $x=0.045$ samples different types of glasses.

this sample occurs at rates of order of the measuring frequency $(50 \mathrm{~Hz})$. Second, the data obtained in cooling in zero applied field at a rate of $20 \mathrm{mK} / \mathrm{h}$ differ from those obtained on warming. In particular, both $\chi^{\prime}$ and $\chi^{\prime \prime}$ peak at higher temperatures for increasing $T$; note that even after entering the warming part of a thermal cycle $\chi^{\prime}$ and $\chi^{\prime \prime}$ continue to decrease. Finally, the peaks in $\chi^{\prime}$ and $\chi^{\prime \prime}$ are at only $\frac{2}{3}$ the demagnetizing limit. ${ }^{11}$

For $x=0.045$ [Fig. 1 (c)], $\chi^{\prime}$ and $\chi^{\prime \prime}$ are again of almost the same size. However, in contrast to what occurs for $x=0.167$, the warming and cooling curves coincide when $T$ is changed at rates less than $20 \mathrm{mK} / 2 \mathrm{~h}$. Furthermore, both $\chi^{\prime}$ and $\chi^{\prime \prime}$ vanish as $T$ approaches zero; thus, at low temperatures essentially all the spins in this sample are frozen. The maximal values of $\chi^{\prime}$ and $\chi^{\prime \prime}$ are well below the demagnetizing limits, ${ }^{11}$ so that the peaks do not correspond to the onset of ferromagnetic order. Note also that $0.2 \mathrm{~K}$ is almost three times as large as $0.045 T_{c}(x=1)$ $=0.069 \mathrm{~K}$. Therefore, glassy behavior preempts ferromagnetism in this sample. Data taken at other frequencies and shown in Fig. 2 provide further evidence for a glasslike magnetic response in $\mathrm{LiHo}_{x} \mathrm{Y}_{1-x} \mathrm{~F}_{4}$ with $x=0.045$. As $T$ is increased, the $\chi^{\prime}(T)$ curves begin to overlap for progressively higher frequencies. Concomitantly, as $f$ is reduced, the maximum in $\chi^{\prime}(T)$ moves to lower $T$. The inset shows the locus of the maxima in the $\log _{10} f-1 / T$ plane. For the two decades of frequency probed, a straight line corresponding to the Arrhenius law,

$$
1 / f=\tau_{\mathrm{char}}=\tau_{0} \exp \left(E_{B} / k_{B} T\right),
$$

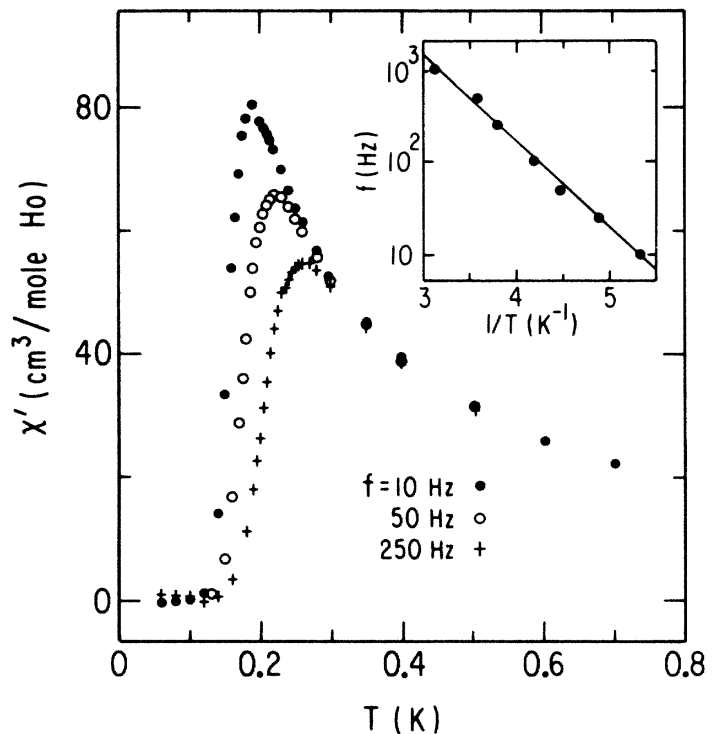

FIG. 2. Frequency $f$ dependence of $\chi^{\prime}(T)$ for $x=0.045$. The temperature where $\chi^{\prime}$ peaks follows an Arrhenius law as a function of $f$ (inset), typical of glassy behavior.

with $\tau_{0}=1.0 \times 10^{-6} \mathrm{sec}$ and $E_{B} / k=2.2 \mathrm{~K}$, describes the data well. The extraordinarily long characteristic time $\tau_{0}$ presumably accounts for the observtion of hysteresis ${ }^{13}$ in the ac susceptibility: From Eq. (1), $\tau_{\text {char }}=1 \mathrm{~h}$ for $T=0.1$ $\mathrm{K}$.

The physically reasonable values of the fit parameters lend credence to the validity of the Arrhenius form. This is in contrast to susceptibility studies ${ }^{14}$ of manganese amorphous aluminosilicate, an insulating spin glass with short-range magnetic interactions, where a fit to the Arrhenius law results in completely unphysical parameters. Extension of the experiment on $\mathrm{LiHo}_{0.045} \mathrm{Y}_{0.955} \mathrm{~F}_{4}$ to lower $f$ could, of course, reveal deviations from Arrhenius behavior.

In order to further elucidate the nature of the low temperature states of the $x=0.045$ and 0.167 samples, we have measured the approach to equilibrium in $\chi^{\prime}$ after warming to $T_{1}=160 \mathrm{mK}$ from $T_{0}=100 \mathrm{mK}$, at which temperature the sample previously had been held after cooling from $0.7 \mathrm{~K}$ in an applied field $H_{\text {cool }}$ of either zero or $6 T$ (see the inset of Fig. 3). For $x=0.045$, we find that within $2 \mathrm{~h}, \chi^{\prime}\left(T_{1}\right)$ approaches the value obtained on cooling to $T_{1}$. This result is independent of $H_{\text {cool }}$, which implies that at $T=160 \mathrm{mK}$, the ferromagnetic state is unstable; note that $g \mu_{\mathrm{eff}} H$ for $H=6 T$ and $\mu_{\mathrm{eff}}=7 \mu_{B}$ corresponds to a temperature of $56 \mathrm{~K} \gg T_{c}(x=1)$. Indeed, we find by the same method that for all $T_{1}>T_{0}=10 \mathrm{mK}$, the ferromagnetic state is unstable. As shown in Fig. 3, matters are very different for $x=0.167$. First, the equilibration times exceed $10 \mathrm{~h}$, a result which accounts for the continued decrease in $\chi^{\prime}(T)$ and $\chi^{\prime \prime}(T)$, described above [see Fig. 1(b)], after the onset of warming in a thermal cycle. Second, $\chi^{\prime}(T)$ converges to equilibrium values that depend on $H_{\text {cool }}$. In particular, $\chi^{\prime}\left(H_{\text {cool }}=6 T\right)$ decreases from a high value to a value lower than the final value toward which $\chi^{\prime}\left(H_{\text {cool }}=0\right)$ increases. Thus, immediately 


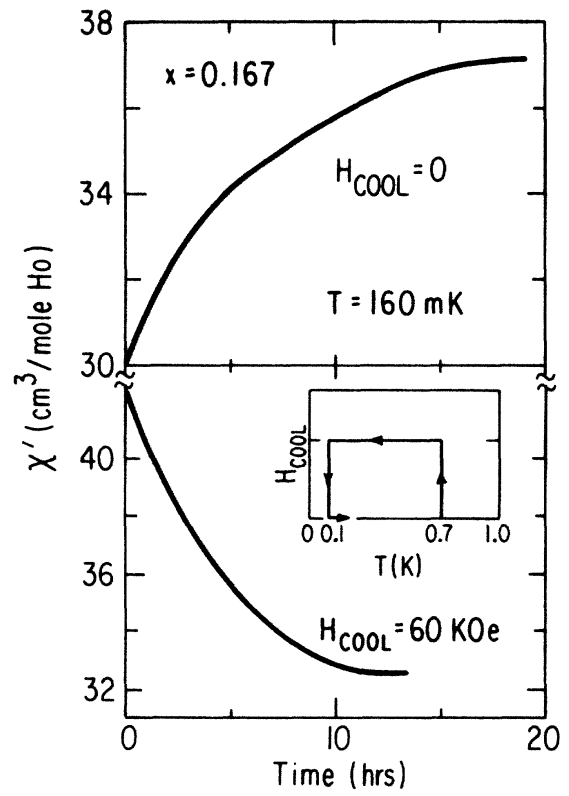

FIG. 3. The long relaxation times in the ac susceptibility $(f=50 \mathrm{~Hz})$ and the contrasting behavior for the field-cooled and zero-field-cooled cases for $x=0.167$ demonstrate the existence of different metastable states. The inset diagrams the field and thermal history $(10-\mathrm{mK} / \mathrm{min}$ cooling rate, $8-\mathrm{h}$ equilibration at $100 \mathrm{mK}, 20-\mathrm{mK} / \mathrm{h}$ warming rate, then relaxation).

after reaching $T_{1}$, the field-cooled state has more spins in clusters with low barriers to relaxation, as one might expect if the zero-field-cooled state were closer to the "true" ground state. Upon relaxation, however, there are fewer moveable spins after field cooling than after zero-field cooling. The field-cooled state evolves toward a configuration where larger numbers of spins are locked together, most probably in ferromagnetic clusters.

We have presented the first magnetic susceptibility data on a model random dipolar coupled system, $\mathrm{LiHo}_{x} \mathrm{Y}_{1-x} \mathrm{~F}_{4}$. Three qualitatively different types of behavior are found. For large $x(0.46), \mathrm{LiHo}_{x} \mathrm{Y}_{1-x} \mathrm{~F}_{4}$ undergoes a conventional ferromagnetic transition at $T=x T_{c}(x=1)$. On the other hand, for small $x(0.045)$, the system behaves as a nearly ideal magnetic glass, with no evidence for a finite-temperature phase transition. Measurements performed after aligning all moments in a strong external field show that the ferromagnetic state is unstable with respect to this glassy state for temperatures as low as $0.01 \mathrm{~K}$. Finally, for intermediate $x(0.167)$, we find extraordinarily long thermal relaxation times for the ac susceptibility, even in zero applied field. In contrast to what occurs for $x=0.045$, the field-cooled, ferromagnetically aligned state gives way to a different final configuration than does the zero-field-cooled state. Thus, $\mathrm{LiHo}_{x} \mathrm{Y}_{1-x} \mathrm{~F}_{4}$ is a model system which will enable us to study the evolution from a simple glass, whose dynamics are dominated by activation over fixed barriers, to a more correlated state of the type commonly associated with spin glasses. It remains to be seen whether this evolution is gradual and a consequence of the approach to ferromagnetism as $x$ is increased, or if there is a well-defined glass-spin-glass multicritical point at a specific $x$ and $T=0$.

We thank R. N. Bhatt, D. Huse, G. F. Mazenko, S. R. Nagel, and A. Ogielski for helpful discussions. Two of us (T.F.R. and G.A.) are grateful to the Aspen Center of Physics where part of the research was performed. The work at The University of Chicago was supported by the National Science Foundation Materials Research Laboratory under Grant No. 82-16892 and a Joseph H. DeFrees Grant from Research Corporation. One of us (T.F.R.) acknowledges support from the Alfred P. Sloan Foundation.
${ }^{1}$ J. M. Luttinger and L. Tisza, Phys. Rev. 70, 954 (1946).

${ }^{2}$ M. J. Stephen and A. Aharony, J. Phys. C 14, 1665 (1981).

${ }^{3}$ A. Aharony, Phys. Rev. B 13, 2092 (1976).

${ }^{4}$ C. Keller and H. Schmutz, J. Inorg. Nucl. Chem. 27, 900 (1965).

${ }^{5}$ D. Belanger, G. Aeppli, J. Axe, and H. Guggenheim (unpublished). For example, the upper bound for the FWHM of the $(1.98,0,0)$ peak in an $x=0.67$ ferromagnetic sample with $T_{c}=0.98 \mathrm{~K}$ is $10 \mathrm{mK}$ (even disregarding finite $q$ broadening and limitations due to instrumental resolution). Hence, concentration fluctuations must be less than $1 \%$.

${ }^{6}$ P. E. Hansen, T. Johansson, and R. Nevald, Phys. Rev. B 12, 5315 (1975).

${ }^{7}$ G. Ahlers, A. Kornblit, and H. J. Guggenheim, Phys. Rev. Lett. 34, 1227 (1975); J. Als-Nielsen, ibid. 37, 1161 (1976); J. A. Griffin, M. Huster, and R. J. Folweiler, Phys. Rev. B 22, 4370 (1980).

${ }^{8}$ P. Beauvillain, J. Seiden, and I. Laursen, Phys. Rev. Lett. 45, 1362 (1980); J. A. Griffin, J. J. Folkins, and D. Gabbe, ibid. 45, 1887 (1980).

${ }^{9}$ R. W. Youngblood, G. Aeppli, J. D. Axe, and J. A. Griffin, Phys. Rev. Lett. 49, 1724 (1982).
${ }^{10}$ J. F. Schooley, G. A. Evans, Jr., and R. J. Soulen, Jr., Cryogenics 20, 193 (1980); R. J. Soulen, Jr., J. F. Schooley, and G. A. Evans, Jr., Rev. Sci. Instrum. 44, 1537 (1973).

${ }^{11} \mathrm{We}$ approximate the shape of the samples by prolate ellipsoids of revolution. The ratio of the major to the minor axes is 1.4 , 1.7 , and 1.8 for the $x=0.46,0.167$, and 0.045 samples, respectively. By this approximation, we find $\chi$ of the $x=0.46$ sample at $93 \%$ of its demagnetizing limit, $\chi$ of the $x=0.167$ sample at $67 \%$ of its demagnetizing limit, and $\chi$ of the $x=0.045$ sample at $21 \%$ of its demagnetizing limit. See A. M. Morrish, The Physical Principles of Magnetism (Wiley, New York, 1965), Appendix II.

${ }^{12}$ See, for example, G. Aeppli, S. M. Shapiro, H. Maletta, R. J. Birgeneau, and H. S. Chen, J. Appl. Phys. 55, 1628 (1984); P.-Z. Wong, S. von Molnar, T. T. M. Palstra, J. A. Mydosh, H. Yoshizawa, S. M. Shapiro, and A. Ito, Phys. Rev. Lett. 55, 2043 (1985).

${ }^{13}$ L. Lundgren, P. Svedlindh, P. Norblad, and O. Beckman, Phys. Rev. Lett. 51, 911 (1983).

${ }^{14}$ P. Beauvillain, C. Dupas, J. P. Renard, and P. Veillet, Phys. Rev. B 29, 4086 (1984). 